\title{
Schizophrenia shows a unique metabolomics signature in plasma
}

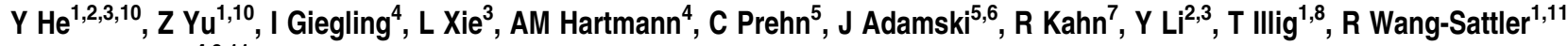 \\ and $D$ Rujescu ${ }^{4,9,11}$
}

Schizophrenia is a severe complex mental disorder affecting $0.5-1 \%$ of the world population. To date, diagnosis of the disease is mainly based on personal and thus subjective interviews. The underlying molecular mechanism of schizophrenia is poorly understood. Using targeted metabolomics we quantified and compared 103 metabolites in plasma samples from 216 healthy controls and 265 schizophrenic patients, including 52 cases that do not take antipsychotic medication. Compared with healthy controls, levels of five metabolites were found significantly altered in schizophrenic patients ( $P$-values ranged from $2.9 \times 10^{-8}$ to $2.5 \times 10^{-4}$ ) and in neuroleptics-free probands ( $P$-values ranging between 0.006 and 0.03 ), respectively. These metabolites include four amino acids (arginine, glutamine, histidine and ornithine) and one lipid (PC ae C38:6) and are suggested as candidate biomarkers for schizophrenia. To explore the genetic susceptibility on the associated metabolic pathways, we constructed a molecular network connecting these five aberrant metabolites with 13 schizophrenia risk genes. Our result implicated aberrations in biosynthetic pathways linked to glutamine and arginine metabolism and associated signaling pathways as genetic risk factors, which may contribute to patho-mechanisms and memory deficits associated with schizophrenia. This study illustrated that the metabolic deviations detected in plasma may serve as potential biomarkers to aid diagnosis of schizophrenia.

Translational Psychiatry (2012) 2, e149; doi:10.1038/tp.2012.76; published online 14 August 2012

\section{Introduction}

Schizophrenia is a severe chronic brain disorder with symptomatic onset in early adulthood and persists throughout life. ${ }^{1}$ It affects approximately $0.5-1 \%$ of the world population ${ }^{2}$ with high heritability. ${ }^{3}$ Currently, the diagnosis of schizophrenia is still merely based on interview of the person and family members as there are no reliable medical diagnostic tests available. ${ }^{4}$ Therefore, the identification of blood markers for schizophrenia has become a crucial issue, which would not only improve our understanding of the disease, but also help diagnose the disease in the early-onset phase and lead to efficient and specific therapies.

Genetic factors and gene-environment interactions contribute largely the liability to the disease. ${ }^{5}$ Family, twin and adoption studies have demonstrated that the rising risk of schizophrenia in relatives relate to strong genetic factors. ${ }^{3}$ A number of genome-wide association studies ${ }^{6-11}$ have conferred that common variations ${ }^{8,9}$ and individually rare structural variants ${ }^{10,11}$ would increase the disease risk. Environmental factors such as living environment, drug abuse and childhood trauma ${ }^{12}$ also have important roles in the pathogenesis of this disease.
Recently, several metabolomic studies also demonstrated metabolic abnormalities in schizophrenia. Holmes ${ }^{13}$ used ${ }^{1} \mathrm{H}$ NMR spectroscopy to measure the metabolic profiles of 152 cerebrospinal fluid samples from drug-naïve patients compared with matched controls and found elevated glucose concentrations in patients, whereas serum glucose level showed no difference. Kaddurah-Daoku et al. ${ }^{14}$ compared lipid levels in 27 schizophrenic patients with 16 healthy controls and revealed significantly decreased levels in patients' plasma. Another Finland population study performed serum metabolome of 45 cases and controls each on a lipidomics platform and a small polar metabolites platform. ${ }^{15}$ They observed that six lipid clusters mainly including saturated triglycerides and two small-molecular clusters containing glutamic acid and proline were elevated in schizophrenia, which suggested the relevance between glucoregulatory, proline metabolism and this disease. A recent research ${ }^{16}$ reported a global metabolic profiling study on 112 schizophrenia and 110 controls using serum and urine samples. The study detected the potential metabolite markers consisting of several fatty acids and ketone bodies, and they presumed the upregulated fatty acid catabolism may result

\footnotetext{
${ }^{1}$ Research Unit of Molecular Epidemiology, Helmholtz Zentrum München, Neuherberg, Germany; ${ }^{2}$ Key Laboratory of Systems Biology, Chinese Academy of Sciences, Shanghai, China; ${ }^{3}$ Shanghai Center for Bioinformation Technology, Shanghai, China; ${ }^{4}$ Department of Psychiatry and Psychotherapy, Ludwig-Maximilians-University, Munich, Germany; ${ }^{5}$ Institute of Experimental Genetics, Genome Analysis Center, Helmholtz Zentrum München, Neuherberg, Germany; ${ }^{6}$ Department of Experimental Genetics, Technische Universität München, Munich, Germany; ${ }^{7}$ Department of Psychiatry, University Medical Center Utrecht, Utrecht, The Netherlands; ${ }^{8}$ Hannover Unified Biobank, Hannover Medical School, Hannover, Germany and ${ }^{9}$ Department of Psychiatry, University of Halle, Germany

Correspondence: Dr R Wang-Sattler, Research Unit of Molecular Epidemiology, Helmholtz Zentrum München, Neuherberg 85764, Germany.

E-mail: rui.wang-sattler@helmholtz-muenchen.de

${ }^{10}$ Joint first authors.

${ }^{11}$ Joint senior authors.

Keywords: metabolic pathway; metabolomics; neuroleptics-free; schizophrenia

Received 27 June 2012; revised 7 July 2012; accepted 7 July 2012
} 
from an insufficiency of glucose supply in the brains of schizophrenia. These studies discovered various metabolite signatures and provided valuable clues to the understanding of the disease mechanism; however, they contained relative small numbers of individuals and thus limit their abilities to identify the diagnostic signatures in the disorder.

To identify the metabolite alteration in schizophrenia, which may serve as potential biomarker signatures, we targeted 103 metabolites, including amino acids, acylcarnitines, glycerophospholipids, sphingolipids and hexose, to analyse the plasma metabolite-concentration changes between 265 patients with schizophrenia and 216 healthy individuals.

\section{Materials and methods}

\section{Study population}

Schizophrenic patients. We recruited patients ascertained with schizophrenia from the Munich area in Germany. Case participants had a DSM-IV and ICD-10 diagnosis of schizophrenia with the following subtypes: paranoid $75.6 \%$ disorganized $17.3 \%$, catatonic $2.3 \%$ and undifferentiated $4.9 \%$. Detailed medical and psychiatric histories were collected, including the Structured Clinical Interview for DSM-IV, to evaluate lifetime Axis I and II diagnoses. ${ }^{17,18}$ We excluded patients with a history of head injury or neurological diseases. All cases were outpatients or stable in-patients. Two hundred and sixty-six patients, aged from 19 to 67 years old, were included in this study. Eighty percentage of them are undergoing treatment with stable doses of one of the neuroleptics (haloperidol, $n=26$; amisulpride, $n=37$; clozapine, $n=38$; olanzapine, $n=37$; quetiapine, $n=38$ and risperidone, $n=38$ ). Age at onset was 24.3 (8.6) years old, $18.8 \%$ of the patients had a schizophrenia diagnosis in first-degree relatives, $31.2 \%$ in relatives in general.

Healthy individuals. Healthy volunteers were randomly selected from the general population of Munich, Germany and contacted by mails. We excluded subjects with central neurological diseases and psychotic disorders or subjects who had first-degree relatives with psychotic disorders by several screenings ${ }^{17-20}$ (for detailed exclusion criterion, see Braunewell et al. ${ }^{21}$ ). Finally, 224 control subjects, 20-67 years of age, were included in this study.

This study was approved by the local ethics committee and conducted according to the ethical standards laid down in the Declarations of Helsinki. All subjects gave their written informed consent. Detailed depictions of all subjects were published elsewhere. ${ }^{21}$

Plasma samples. Blood (approximately $7.5 \mathrm{ml}$ ) was obtained from forearm vein and drawn in EDTA-containing tubes for biomarker studies. ${ }^{22}$ The samples were centrifuged for $10 \mathrm{~min}$ at $4{ }^{\circ} \mathrm{C}$ and the resulting plasma aliquoted into Eppendorf tubes, which were frozen immediately at $-80^{\circ} \mathrm{C}$.

Metabolite measurements. All plasma samples were measured by the commercially available AbsoluteIDQ p150 kit (BIOCRATES Life Sciences AG, Innsbruck, Austria), which allows simultaneous quantification of 163 metabolites using flow injection analysis mass spectrometry. This method has been proven to be in conformance with FDA-Guidelines 'Guidance for Industry-Bioanalytical Method Validation (May 2001)', which implies proof of reproducibility within a given error range. The procedures of this assay were described in more details elsewhere. ${ }^{23-26}$

Metabolite measurements for all 490 samples were performed in three batches. All sample analyses underwent quality assurance and control procedures. Nine samples were excluded for further analysis as they had missing values. Ten replicate samples were measured across the three batches, and concentration of each metabolite was adjusted based on that to minimize potential batch effects.

To ensure data quality, each metabolite had to go through following stringent criteria, which we applied previously ${ }^{25}$ : (1) average value of the coefficient of variance for the metabolite in three quality control samples, which represents mixed human plasma samples provided by the manufacturer, should be $<25 \%$. (2) $90 \%$ of all measured sample concentrations for the metabolite should be above the limit of detection. (3) The correlation coefficient of the metabolite between measurements in ten duplicate samples should exceed 0.5. In total, 103 metabolites passed the three criteria, and the final metabolite panel contained 13 amino acids, 8 acylcarnitines, 60 phosphatidylcholines (PCs), 8 lysoPCs, 13 sphingomyelins and total hexoses $(\mathrm{H} 1)$. Concentrations of all analyzed metabolites were reported in $\mu \mathrm{m}$. Characteristics and average values of all measured metabolites were summarized in Supplementary Table 1.

Statistics analysis. The design and analysis strategy were depicted in Supplementary Figure S1. Multivariate logistic regression analysis was used to identify the metabolites with significant concentration difference between schizophrenic patients (cases) and healthy controls. Odds ratios for single metabolites were calculated between two groups. We normalized concentrations of each metabolite to have a mean of zero and a s.d. of one for the working data set. Age, sex and body mass index (BMI) were added to the logistic regression analysis as covariates. The significance level was adjusted using the Bonferroni correction to $4.8 \times 10^{-4}(0.05 / 103)$.

To exclude the metabolites with significant concentration changes influenced by neuroleptics-taken (NT), one-way ANOVA test coupled with Tukey's honestly significant difference test ${ }^{27}$ were conducted on the metabolites, which

Table 1 Demographic data of schizophrenic cases and healthy controls

\begin{tabular}{|c|c|c|c|}
\hline & \multicolumn{2}{|c|}{ Cases } & \multirow[t]{2}{*}{ Controls } \\
\hline & $N F$ & $N T$ & \\
\hline $\begin{array}{l}\text { Total number }(N) \\
\text { Sex (male) } \\
\text { Age (mean } \pm \text { s.d.) } \\
\text { BMI (mean } \pm \text { s.d.) }\end{array}$ & $\begin{array}{c}\quad 52 \\
55.8 \% \\
39.3 \pm 11.2 \\
24.9 \pm 4.8\end{array}$ & $\begin{array}{c}\quad 213 \\
62.0 \% \\
36.9 \pm 11.7 \\
26.4 \pm 4.9\end{array}$ & $\begin{array}{c}216 \\
51.9 \% \\
38.9 \pm 10.6 \\
25.3 \pm 4.4\end{array}$ \\
\hline
\end{tabular}

Abbreviations: BMI, body mass index; NF, neuroleptics-free; NT, neurolepticstaken. 
Table 2 Significantly different metabolites in schizophrenia and test for neuroleptics influence

\begin{tabular}{|c|c|c|c|c|}
\hline \multirow[t]{2}{*}{ Metabolites } & \multicolumn{2}{|c|}{$\begin{array}{c}\text { Cases versus } \\
\text { controls }(N F+N T \text { versus } \\
\text { controls) }\end{array}$} & \multirow{2}{*}{$\begin{array}{c}\text { NF versus } \\
\text { controls } \\
\text { P-value }\end{array}$} & \multirow{2}{*}{$\begin{array}{l}\text { NT versus } \\
\quad N F \\
\text { P-value }^{b}\end{array}$} \\
\hline & OR $(95 \% C l)$ & $\mathrm{P}$-value & & \\
\hline Ornithine $^{c}$ & $1.92(1.53-2.44)$ & $2.9 \times 10^{-8}$ & 0.01 & 0.41 \\
\hline Glutamine $^{c}$ & $0.57(0.46-0.7)$ & $1.3 \times 10^{-7}$ & 0.006 & 0.98 \\
\hline Arginine ${ }^{c}$ & $0.64(0.52-0.78)$ & $9.1 \times 10^{-6}$ & 0.02 & 0.99 \\
\hline Histidine $^{c}$ & $0.7(0.58-0.84)$ & $2.5 \times 10^{-4}$ & 0.03 & 0.82 \\
\hline PC ae C38: $6^{\mathrm{C}}$ & $0.59(0.46-0.73)$ & $4.0 \times 10^{-6}$ & 0.03 & 0.66 \\
\hline$P C$ ae $C 34: 3^{d}$ & $0.65(0.52-0.8)$ & $1.2 \times 10^{-4}$ & 0.5 & 0.04 \\
\hline $\mathrm{C} 2$ & $0.66(0.53-0.81)$ & $1.2 \times 10^{-4}$ & 0.16 & 0.5 \\
\hline Methionine & $0.7(0.57-0.84)$ & $3.0 \times 10^{-4}$ & 0.13 & 0.94 \\
\hline $\mathrm{PC}$ ae $\mathrm{C} 36: 5$ & $0.57(0.45-0.7)$ & $7.2 \times 10^{-7}$ & 0.08 & 0.36 \\
\hline $\mathrm{PC}$ ae $\mathrm{C} 42: 1$ & $1.67(1.32-2.16)$ & $4.5 \times 10^{-5}$ & 0.74 & 0.07 \\
\hline PC ae C38.5 & $0.69(0.55-0.84)$ & $4.0 \times 10^{-4}$ & 0.16 & 0.88 \\
\hline PC aa C38:0 & $0.67(0.53-0.82)$ & $2.2 \times 10^{-4}$ & 0.08 & 0.84 \\
\hline
\end{tabular}

Abbreviations: $\mathrm{Cl}$, confidence interval; NF, neuroleptics-free cases; NT, neuroleptics-taken cases; OR, odds ratio.

a Odds ratios (95\% confidence intervals) and $P$-values were calculated from multivariate logistic regression analysis with adjustment for age, sex and BMI (Bonferroni correction $P$-value $<4.8 \times 10^{-4}$ ). ' Tukey's HSD test was applied to test concentration difference between the two groups. $P$-value $<0.05$ are shown in bold. "No significant neuroleptics influence (that is no significan difference in NT versus NF, but difference in NF versus controls). ${ }^{\text {dPotential }}$ neuroleptics influence (that is, significant difference in NT versus NF; however, no difference in NF versus controls). were significant in logistic regression. Multiple comparisons were performed between NT cases and neuroleptics-free (NF) cases, as well as between NF versus controls. The metabolites were categorized into three following groups: (1) No significant neuroleptics influence (that is metabolites showed no significant difference in NT-NF comparison, but they differed in NF versus controls.) (2) Potential neuroleptics influence (that is metabolites showed significant difference in NT versus NF comparison, whereas they showed no difference in NF versus controls.) (3) Unknown (that is the metabolites did not show significant difference in either comparison.). We kept the metabolites with no significant neuroleptics influence for further analysis, and excluded those in (2) and (3) groups.

To evaluate the performance of the five metabolites, we randomly split the participants into detection and validation groups for one hundred times to cross validate our classification model. Each time, we randomly chose $90 \%$ participants for model estimation, and used the remaining $10 \%$ samples for validation. The receiver-operating characteristic curve indicated the power of discrimination between the cases and controls with $95 \%$ confidence intervals s.d. of the true-positive rate. All calculations were performed under the $\mathrm{R}$ statistical environment (http://www.r-project.org/).

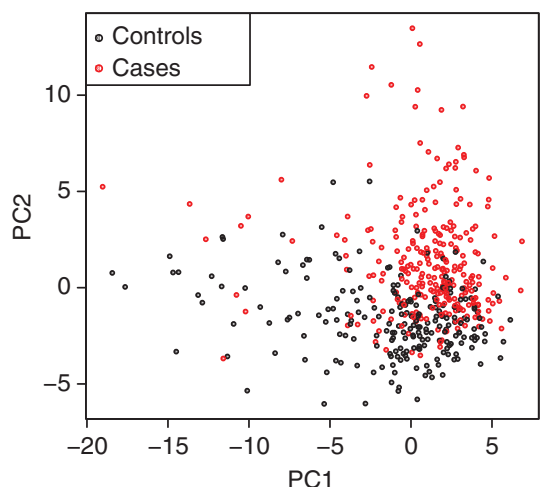

C

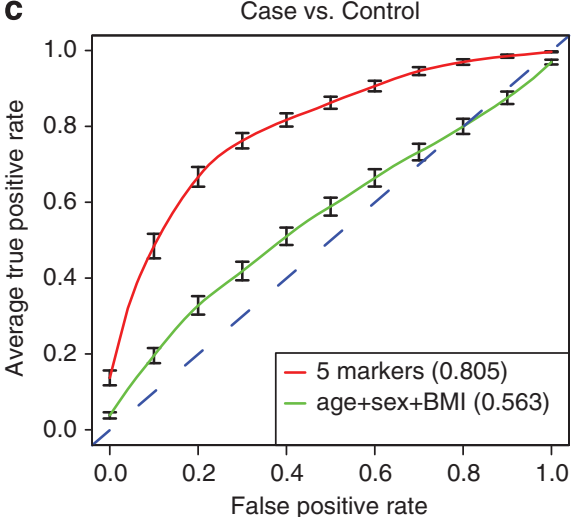

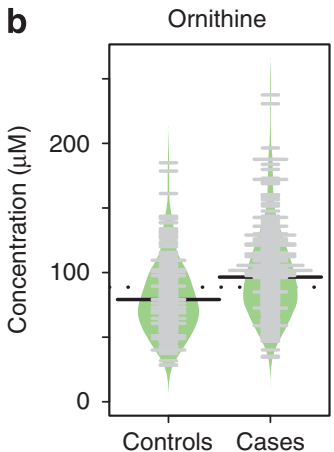
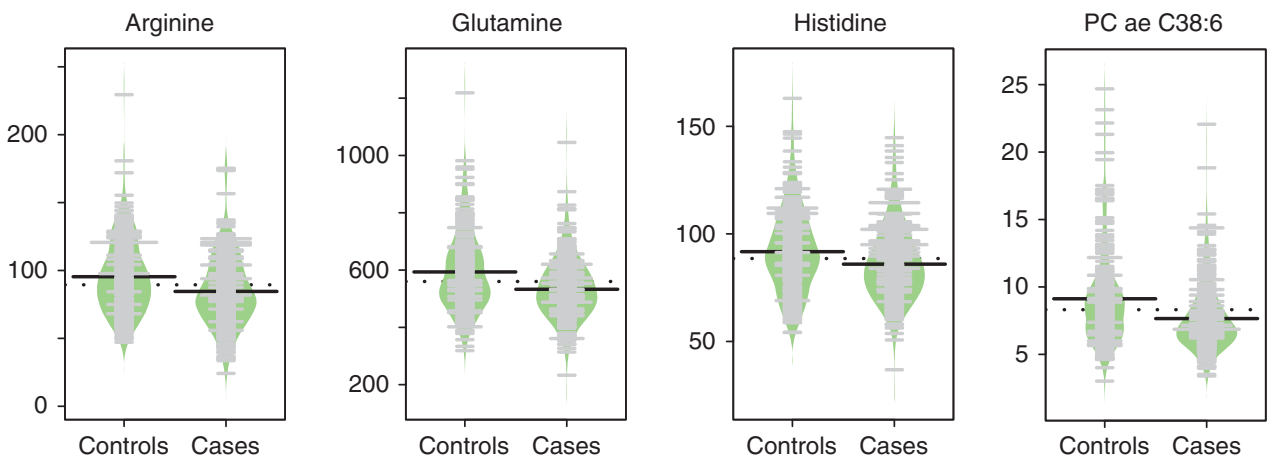

Figure 1 Metabolomics discriminate schizophrenic patients from healthy individuals. (a) Partial least square (PLS) analysis demonstrate a clear separation of metabolite concentration between patients with schizophrenia (red) and healthy individuals (black). (b) The concentrations of five metabolite signatures were shown in beanplots, which provide information on the mean level (solid black line), individual data points (short gray lines) and the density of the distribution between controls and cases. (c) The receiveroperating characteristic (ROC) curves indicated the effect of discrimination based on the combination of five metabolite signatures in the model (red) and the combination of age, sex and BMI (green) with $95 \%$ confidence intervals s.e. of the true-positive rate. 


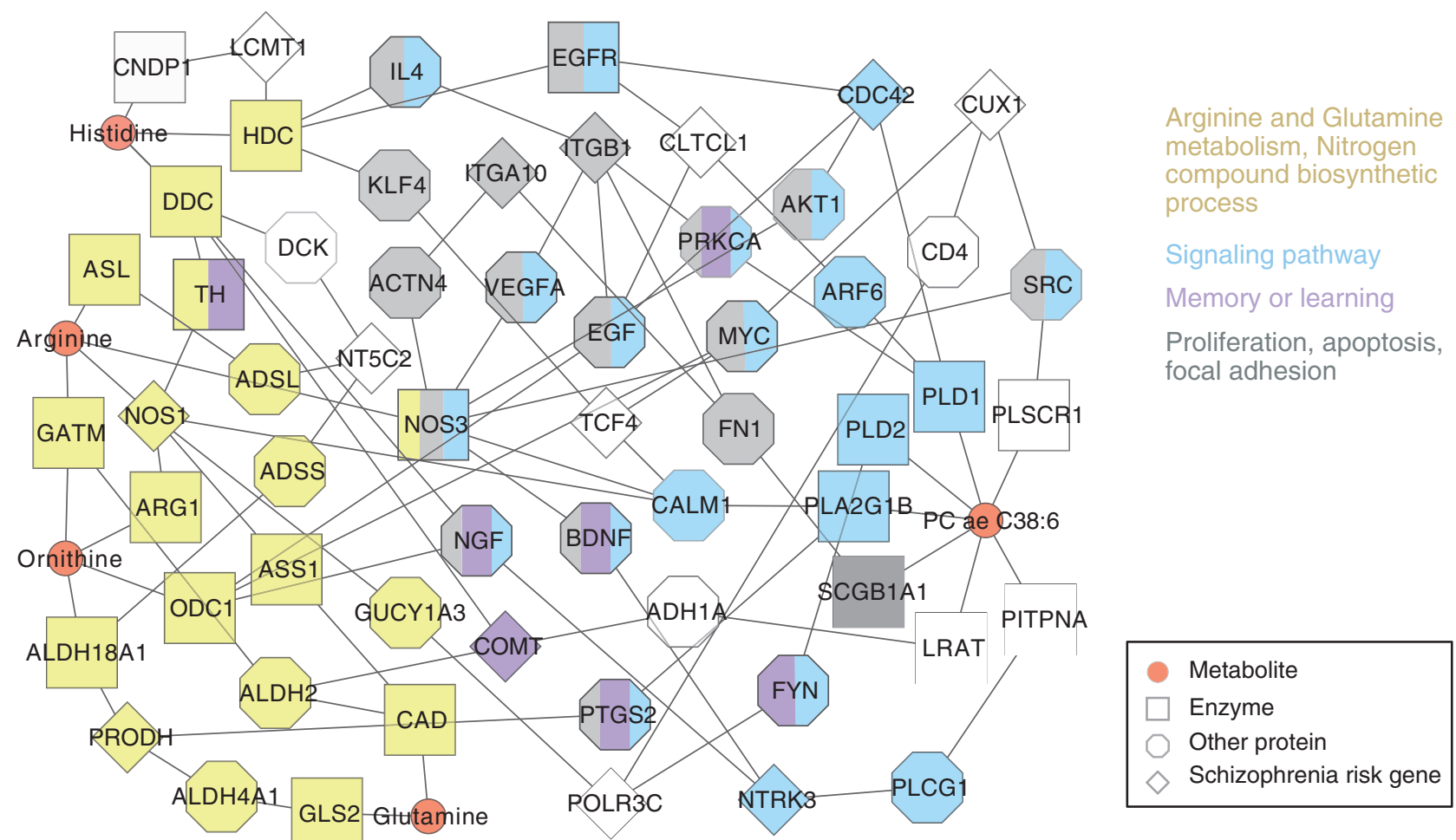

Figure 2 Concept of molecular network in schizophrenia. The network showed associations between the five candidate metabolites and the schizophrenia risk genes. For details, see Supplementary Table 2. Node shaped as circle, square, octagon and diamond represent metabolite, enzyme, intermediate protein and schizophrenia risk gene, respectively. Nodes overlaid with a color key representing their major functions: arginine, glutamine metabolism process and nitrogen compound biosynthetic process (yellow); signaling pathway (blue); memory or learning (purple); proliferation, apoptosis and focal adhesion (gray).

Network analysis. We collected 152 schizophrenia risk genes from the latest genome-wide association studies (Supplementary Table 2). 6,7,9,10,28-33 Schizophrenia molecular network was constructed by connecting candidate metabolites, enzymes, intermediate proteins and schizophrenia risk genes. A background network was structured based on the metabolite-enzyme interaction from the Human Metabolome Database $^{34}$ (HMDB) and the protein functional association from the Search Tool for the Retrieval of Interacting Genes/ Proteins (STRING) database ${ }^{35}$ (STRING score at least 0.7). Using the metaP-Server ${ }^{36}$ we mapped the candidate metabolites to HMDB IDs, and derived their associated enzymes according to the annotations. We first mapped the candidate metabolites and their associated enzymes to the background network, and then connected the enzymes with the schizophrenia risk genes allowing for one intermediate protein in the paths. The paths from enzymes to risk genes were searched by the shortest path algorithm implemented in the igraph library (http://igraph.sourceforge.net/index.html), which finds the appropriate paths with the highest scores. The network consist of nodes (metabolites, enzymes, intermediate proteins and risk genes) and edges (associations and connections), and visualized by Cytoscape (http://www.cytoscape.org/). We used Database for Annotation, Visualization and Integrated Discovery (DAVID) ${ }^{37}$ to analyze the enriched biological function and involved pathway of the genes and proteins in the network based on Gene Ontology ${ }^{38}$ and Kyoto Encyclopedia of Genes and Genomes ${ }^{39}$ annotations under Bonferroni-corrected $P$-value $<0.05$.

\section{Results}

Schizophrenic patients separated from healthy individuals. After the quality controls, a total of 481 individuals and 103 metabolites were covered in the final data set. Among them, 216 subjects were healthy controls and 265 subjects were cases diagnosed with schizophrenia, including 52 patients without taking any neuroleptic (Table 1). The cases and controls showed no significant difference in sex, age and BMI demographics.

To explore the global differences between the cases and controls in metabolite concentration levels, a partial least square analysis was carried out on the metabolite profiles. The partial least square result (Figure 1a) demonstrated a separation of metabolite profiles between the two groups, indicating some schizophrenic signatures were captured among the metabolic difference.

Metabolites with significant alteration in schizophrenia. To identify significant metabolite concentration changes between schizophrenic patients and healthy controls, multivariate logistic regression analysis were carried out on each of the 103 metabolites, with concentration as dependent and schizophrenia as explanatory variable besides the covariates sex, age and BMI. Twelve metabolites, including five amino acids, one acetylcarnitine and six PCs (Table 2), displayed significantly different concentrations in schizophrenic subjects compared with controls. 

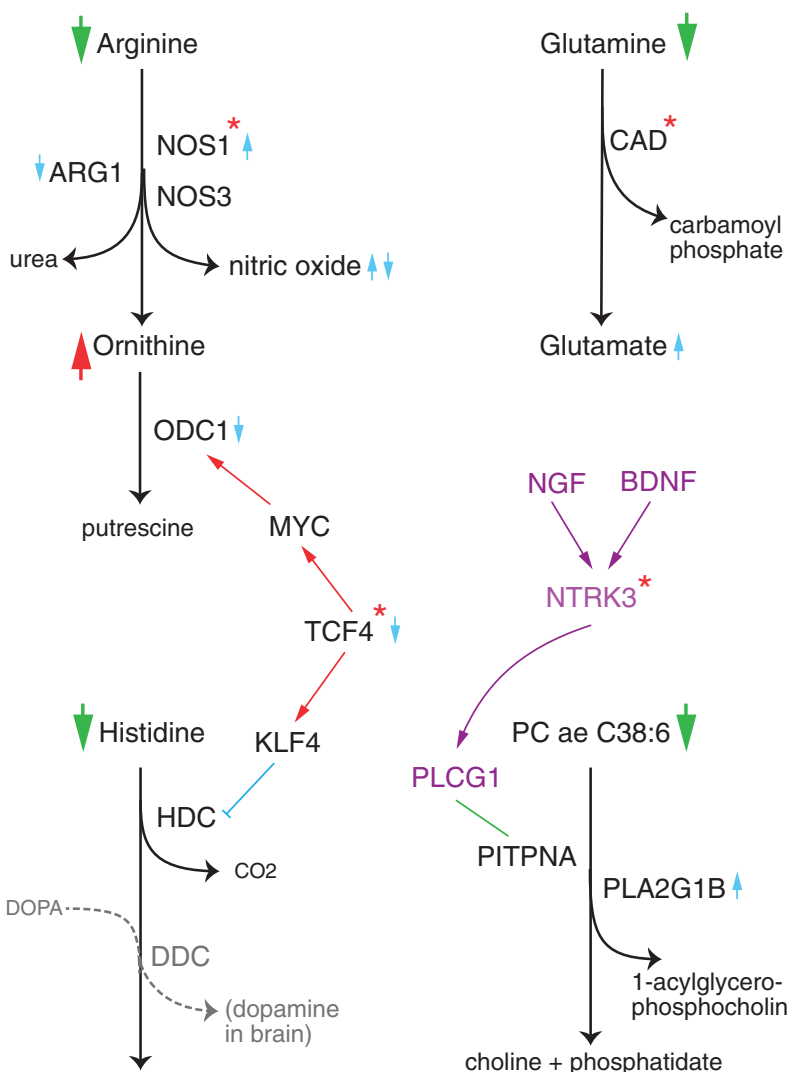

$\downarrow$ Histamine

\begin{tabular}{|c|c|c|}
\hline$\longrightarrow$ & Metabolism pathway & * Schizophrenia risk gene \\
\hline$\rightarrow$ & Activation & \\
\hline & Inhibition & $\mathrm{Ol}$ \\
\hline & Physical interaction & $4 \downarrow$ Reported changes \\
\hline
\end{tabular}

Figure 3 Influence of genetic risks on the metabolic pathways. The schizophrenia risk genes (marked by red stars) are supposed to be involved in the regulation of these metabolic pathways (black). Types of regulations are visualized as following: activation (red), inhibition (blue), neurotrophin signaling (purple) and physical interaction (gray). The observed metabolite concentration changes between schizophrenics and healthy individuals are denoted by the red and green arrows next to them. Blue arrows suggest the possible alteration reported in previous publications. Bigger/smaller arrows indicate reported/observed changes in metabolite concentrations or gene/protein level.

Furthermore, multiple comparisons were conducted between NT and NF, as well as NF and controls, considering the potential neuroleptics influence on these 12 metabolites. We further applied ANOVA test coupled with Turkey's honestly significant difference test and finally identified five metabolites (that is, higher concentration of ornithine and lower concentration of arginine, glutamine, histidine and PC ae C38:6 in schizophrenic patients) showing no significant difference in NT versus NF comparison, but differed in NF versus controls. To visualize these five metabolites, the concentration distributions in cases and controls were displayed in Figure $1 \mathrm{~b}$ by beanplots. ${ }^{40}$ The average area under the receiver-operating characteristic curve value for the five metabolites reached $80.5 \%$, indicating a relatively high sensitivity and specificity for detection of schizophrenia compared with the value of the combination of sex, age and BMI (56.3\%).

Pathways in schizophrenia molecular network. To explore the biological functions and pathways that are related to the significantly changed metabolites, we applied a metabolite-gene/protein bioinformatic approach to construct a schizophrenia-specific molecular network, starting from 152 schizophrenia genetic risk genes to connect with the founded five aberrant metabolites through one intermediate protein and enzyme in the path. Thirteen schizophrenia risk genes (that is nitric oxide synthase 1 (NOS1), transcription factor 4 (TCF4), neurotrophin receptor kinase (NTRK3), catechol-O-methyltransferase (COMT), mitochondrial proline dehydrogenase $(P R O D H)$, leucine carboxyl methyltransferase 1 (LCMT1), cytosolic purine 5'-nucleotidase (NT5C2), DNA-directed RNA polymerase III subunit RPC3 (POLR3C), integrin beta-1 (ITGB1), integrin alpha-10 (ITGA10), clathrin, heavy chain-like 1 (CLTCL1), cell division control protein 42 homolog (CDC42) and cut-like homeobox 1 (CUX1)) were connected to the five metabolites (Figure 2).

Function annotation analysis was performed on the proteins/genes in the network. Sixty biological functions such as glutamine metabolic process, learning or memory, and so on were found significantly involved in the constructed network (Figure 2 and Supplementary Table 3). Arginine, ornithine, glutamine and histidine and their associated proteins/genes were mainly involved in nitrogen compound biosynthetic processes as well as glutamine and arginine metabolism process. PC ae C38:6 and its connecting proteins/genes dominantly participated in several signaling pathways, such as immune-related signaling (for example, Fc epsilon RI signaling and Fc gamma R-mediated phagocytosis, Supplementary Table 3) and neurotrophin signaling.

\section{Discussion}

In our study, we quantified 103 metabolites concentration with 265 schizophrenic patients and 216 healthy individuals. We identified five metabolites (ornithine, arginine, glutamine, histidine and PC ae C38:6), which differed significantly between schizophrenic patients and controls, but are not influenced by antipsychotic medication. Furthermore, using a network approach, the altered five metabolites were associated with 13 schizophrenia risk genes.

Arginine and ornithine. Higher blood concentration of ornithine ${ }^{15,41}$ in schizophrenia is consistent with our observation in plasma; however, elevated arginine concentration ${ }^{41}$ conflicts with ours. A key risk gene NOS1 ${ }^{30}$ is involved in the arginine-nitric oxide pathway, ${ }^{42}$ in which cases NOS compete with arginase (ARG1) for the same substrate L-arginine and produce nitric oxide (NO) (Figure 3). Alteration of NO metabolism has been reported in schizophrenia, ${ }^{43}$ which may impact on processes such as synapses formation, neurotransmission and so on. Increased NOS activity ${ }^{44,45}$ and decreased ARG1 activity ${ }^{42}$ were both reported in plasma samples of schizophrenics. According to previous findings, 
we propose that substrate arginine consumption and downstream ornithine accumulation in the urea cycle may be induced by the aberrant NO metabolism in schizophrenia, although it is not clear whether over- or under-production of NO occurs. ${ }^{42,43}$ Another high-risk gene, TCF4 ${ }^{8,31}$ that has key roles in neurodevelopment may also have impact on these two amino acids. Differential expression studies of TCF4 demonstrated its downregulation in red blood cells and upregulation in cerebellar cortex of schizophrenics. ${ }^{46}$ We assume that the dysfunction of TCF4 in blood may suppress its activation of ornithine decarboxylase 1 (ODC1). Decreased expression of ODC in the ornithine-polyamine metabolism, ${ }^{47}$ which supports our assumption of the suppressed ornithine decarboxylation process, might be responsible for the higher concentration of ornithine in the disease.

Histidine. Histidine is the precursor of the ubiquitous neurohormone neurotransmitter histamine. Opposite to our observation, higher level of histidine was reported in schizophrenic plasma samples. ${ }^{41,48,49}$ As of histamine, lower concentration in whole blood ${ }^{50}$ and higher level in cerebrospinal fluid ${ }^{51}$ occurs in schizophrenic patients. The downregulation of TCF4, as we mentioned above, could probably release its inhibition on histidine decarboxylase (Figure 3), which implies an accelerated histidine decarboxylation process in schizophrenia.

Glutamine. Dysfunction of glutamine-glutamate cycle between neurons and glia was proposed having roles in the pathophysiology of schizophrenia. ${ }^{52-54}$ Lower concentration of glutamine was detected in our schizophrenic plasma samples, whereas elevated glutamate was reported both in serum and cortex of patients. ${ }^{54}$ Nevertheless, the dependency of glutamate concentration between brain and blood seems weak due to the restricted controlled passage across the blood-brain barrier. ${ }^{55}$ A glutamine-dependent carbamoylphosphate synthase enzyme involved in glutamine hydrolysis (Figure 3) was found with a single-nucleotide polymorphismassociated with schizophrenia, ${ }^{28}$ but the detailed mechanism is yet unclear.

Although discussed controversially, it appears that amino-acid alteration occurs in the schizophrenia psychopathology, and the plasma amino-acid concentration changes, to some extent, could reflect the susceptibility to the brain disorder.

Phosphatidylcholines. Cell membrane abnormalities associated with disordered phospholipids composition and metabolism are indicated in schizophrenia pathogenesis. ${ }^{56,57}$ Significantly lower concentrations of PC in schizophrenic postmortem brain tissues ${ }^{58}$ are in agreement with our observation of lower PC levels in schizophrenia. However, several similar studies ${ }^{15,16}$ did not show the significant difference in phospholipids. In Orešič's ${ }^{15}$ study, significantly higher metabolite levels in saturated triglycerides were detected, whereas in Yang's recent report ${ }^{16}$ fatty acids and ketone bodies were found significantly elevated in both the serum and urine sample of the patients. The detected metabolite signatures varied in different studies probably due to sample size, varied samples and different platforms. Orešič et al measured serum samples by a global lipidomics platform and a small polar metabolites platform based on mass spectrometry. Yang et al quantified serum and urine samples using a GC-TOF spectral-based global metabolic profiling. Both studies cover a global metabolic profiling in schizophrenia, while our study focused on a targeted metabolomics profiling mainly covering acylcarnitines, amino acids, hexoses, PCs and sphingomyelins in schizophrenic plasma samples. In addition, deficiencies of plasmalogen (that is, phospholipid with vinyl-ether double bonds in the sn-1 position) were also found in Alzheimer's disease and depression, which could be implicated in abnormal signal transduction associated with learning disability and cognitive deficit. ${ }^{59}$ We found a schizophrenia high-risk gene NTRK3 ${ }^{9}$ involved in neurotrophin signaling pathway may disturb the lipid metabolism in schizophrenia (Figure 3). NTRK3 coupled with its ligands BDNF, NGF and partner protein PLCG1 are all involved in the neurotrophin signaling, which promotes the survival and differentiation of peripheral and central neuron. Variation in NTRK3 may disturb the signaling cascade, affecting the binding between protein PLCG1 and PIPNA, then disturbing the regulation on $P C$ transfer, which leads ultimately to a lower PC concentration as we observed. An alternative explanation could be expected that the increased phospholipase A2 activity in schizophrenia ${ }^{60,61}$ implicates an accelerated breakdown of membrane phospholipids in the disease. $^{62}$ In our network analysis, PC ae C38:6 and its connecting proteins/genes mainly participated in several signaling pathways (for example immune-related signaling and neurotrophin signaling). The neurotrophins have important roles in neural development and are transmitted to intracellular through signaling cascades such as MAPK pathway, in turn leading to regulation of cell differentiation and survival, ultimately impacting higher-order activities such as learning and memory.

We also found that PC ae C34:3 showed no significant difference in NF versus controls, but significantly differed in NT versus NF, indicating a potential antipsychotic effect on its concentration change. An association study in Korean inpatients ${ }^{63}$ was to investigate possible associations between single-nucleotide polymorphisms within ten genes with schizophrenia and response to antipsychotics. ABCB4, one of the enzymes of PC ae C34:3 included in the ten candidate genes, which might involve in the metabolism to neuroleptics and need further investigation.

Limitations. The metabolite panels we used in our study contain mainly lipids, amino acids and carnitines and were unable to cover the whole metabolome and some of the metabolites we measured were not specific enough (for example the detailed structure of the PCs are not clear), which leaves us more area to be explored regarding metabolite concentration alterations in schizophrenia. The participants in our study were only Caucasian, which limits our findings to a more general population. However, even with these limits, we shed some lights on the mechanism of schizophrenia. With a broad coverage of metabolites and population, more signatures might be discovered to help better understand schizophrenia. 


\section{Conclusion}

We identified the underlying molecular signatures in plasma of schizophrenic patients compared with healthy individuals. The aberrant concentrations of metabolites in plasma decipher possible disturbed pathways, including glutamine and arginine metabolism, nitrogen compound biosynthetic process, learning memory behavior, several signaling pathways, may reflect the genetic susceptibility of the disease. Although we encapsulated merely a small picture of metabolites, we believe that metabolomics analyses have huge potential to investigate candidate disease markers that will benefit the diagnostics and therapeutics of schizophrenia in the future.

\section{Conflict of Interest}

The authors declare no conflict of interest.

Acknowledgements. We thank all the participants in the study. We also thank all the staff from Genome Analysis Center, Metabolomics Core Facility, who helped in the sample logistics, data collection and metabolite quantification assays, especially Dr Werner Römisch-Margl, Julia Scarpa, Katharina Sckell and Arsin Sabunchi. The research was kindly funded by EU FP7 grant HEALTH-2009-2.2.1-3/ 242114 (Project OPTiMiSE) and in part supported by grants from the German Federal Ministry of Education and Research (BMBF) to Project-FKZ: 01D012030 and to the German Center Diabetes Research (DZD e.V.)

1. Lewis DA, Lieberman JA. Catching up on schizophrenia: natural history and neurobiology. Neuron 2000; 28: 325-334.

2. Saha $S$, Chant $D$, Welham J, McGrath J. A systematic review of the prevalence of schizophrenia. PLOS Med 2005; 2: e141.

3. Tsuang M. Schizophrenia: genes and environment. Biol Psychiatry 2000; 47: 210-220.

4. van Os J, Kapur S. Schizophrenia. Lancet 2009; 374: 635-645.

5. Tandon R, Keshavan MS, Nasrallah HA. Schizophrenia, "just the facts" what we know in 2008. 2. Epidemiology and etiology. Schizophr Res 2008; 102: 1-18

6. Need AC, Ge D, Weale ME, Maia J, Feng S, Heinzen EL et al. A genome-wide investigation of SNPs and CNVs in schizophrenia. PLoS Genet 2009; 5: e1000373.

7. Nieratschker V, Nöthen MM, Rietschel $M$. New genetic findings in schizophrenia: is there still room for the dopamine hypothesis of schizophrenia? Front Behav Neurosci 4: 23.

8. Stefansson H, Ophoff RA, Steinberg S, Sanders AR, Zheng Y, Pe'er I et al. Common variants conferring risk of schizophrenia. Nature 2009; 460: 744-747.

9. Shi J, Levinson DF, Duan J, Sanders AR, Zheng Y, Pe'er I et al. Common variants on chromosome 6p22.1 are associated with schizophrenia. Nature 2009; 460: 753-757.

10. Stone JL, O'Donovan MC, Gurling H, Kirov GK, Blackwood DH, Corvin A et al. Rare chromosomal deletions and duplications increase risk of schizophrenia. Nature 2008; 455 237-241.

11. Walsh T, McClellan JM, McCarthy SE, Addington AM, Pierce SB, Cooper GM et al. Rare structural variants disrupt multiple genes in neurodevelopmental pathways in schizophrenia. Science 2008; 320: 539-543.

12. Morgan C, Fisher H. Environment and schizophrenia: environmental factors in schizophrenia: childhood trauma-a critical review. Schizophr Bull 2007; 33: 3-10.

13. Holmes E, Tsang TM, Huang JT, Leweke FM, Koethe D, Gerth CW et al. Metabolic profiling of CSF: evidence that early intervention may impact on disease progression and outcome in schizophrenia. PLoS Med 2006; 3: 8, e327.

14. Kaddurah-Daouk R, McEvoy J, Baillie RA, Lee D, Yao JK, Doraiswamy PM et al. Metabolomic mapping of atypical antipsychotic effects in schizophrenia. Mol Psychiatry 2007; 12: 934-945

15. Orešič M, Tang J, Seppänen-Laakso T, Zhao Z, Qi X, Zhou K et al. Metabolome in schizophrenia and other psychotic disorders: a general population-based study. Genome Med 2011; 3: 19

16. Yang J, Chen T, Sun L, Zhao Z, Qi X, Zhou K et al. Potential metabolite markers of schizophrenia. Mol Psychiatry 2011.

17. First MB, Spitzer RL, Gibbon M, Williams BW, Benjamin L. Structured Clinical Interview for DSM-IV Axis II Personality Disorders (SCID-II). Biometrics Research Department, New York State Psychiatric Institute: New York, 1990.

18. First MB, Spitzer RL, Gibbon M, Williams JB. Structured Clinical Interview for DSM-IV Axis I Disorders - Patient Edition (SCID - I/P, Version 2.0). Biometrics Research Department, New York State Psychiatric Institute: New York, 1995
19. Rice JP, Reich T, Bucholz KK, Neuman RJ, Fishman R, Rochberg N et al. Comparison of direct interview and family history diagnoses of alcohol dependence. Alcohol Clin Exp Res 1995; 19: 1018-1023.

20. Folstein MF, Folstein SE, McHugh PR. "Mini-mental state": A practical method for grading the cognitive state of patients for the clinician. J Psychiatr Res 1975; 12: 189-198.

21. Braunewell KH, Dwary AD, Richter F, Trappe K, Zhao C, Giegling I et al. Association of VSNL1 with schizophrenia, frontal cortical function, and biological significance for its gene product as a modulator of cAMP levels and neuronal morphology. Transl Psychiatry 2011; 1: e22.

22. Domenici E, Willé DR, Tozzi F, Prokopenko I, Miller S, McKeown A et al. Plasma protein biomarkers for depression and schizophrenia by multi analyte profiling of case-control collections. PLOS ONE 2010; 5: e9166.

23. Illig T, Gieger C, Zhai G, Römisch-Margl W, Wang-Sattler R, Prehn C et al. A genome-wide perspective of genetic variation in human metabolism. Nat Genet 2010; 42: 137-141.

24. Römisch-Margl W, Prehn C, Bogumil R et al. Procedure for tissue sample preparation and metabolite extraction for high-throughput targeted metabolomics. Metabolomics 2011 $1-10$

25. Mittelstrass K, Ried JS, Yu Z, Krumsiek J, Gieger C, Prehn C et al. Discovery of sexual dimorphisms in metabolic and genetic biomarkers. PLoS Genet 2011; 7: e1002215.

26. Yu Z, Kastenmüller G, He Y, Belcredi P, Möller G, Prehn C et al. Differences between human plasma and serum metabolite profiles. PLOS ONE 2011; 6: e21230.

27. Kirk R. Experimental Design: Procedures for the behavioral sciences. Brooks/Cole Pub Co: Belmont, CA, 1968 179-182.

28. Jia P, Wang L, Meltzer HY, Zhao Z. Common variants conferring risk of schizophrenia: a pathway analysis of GWAS data. Schizophr Res 2010; 122: 38-42

29. Ripke S, Sanders AR, Kendler KS, Levinson DF, Sklar P, Holmans PA et al. Genome-wide association study identifies five new schizophrenia loci. Nat Genet 2011; 43: 969-976.

30. O'Donovan MC, Craddock N, Norton N, Williams H, Peirce T, Moskvina V et al. Identification of loci associated with schizophrenia by genome-wide association and followup. Nat Genet 2008; 40: 1053-1055.

31. Rietschel M, Mattheisen M, Degenhardt F, Brown A, Gustafsson O, Agartz I et al. Association between genetic variation in a region on chromosome 11 and schizophrenia in large samples from Europe. Mol Psychiatry; advance online publication, 12 July 2011 (e-pub ahead of print)

32. Purcell SM, Wray NR, Stone JL, Visscher PM, O'Donovan MC, Sullivan PF et al. Common polygenic variation contributes to risk of schizophrenia and bipolar disorder. Nature 2009; 460: 748-752.

33. Athanasiu L, Mattingsdal M, Kähler AK, Brown A, Gustafsson O, Agartz I et al. Gene variants associated with schizophrenia in a Norwegian genome-wide study are replicated in a large European cohort. J Psychiatr Res 2010; 44: 748-753.

34. Wishart DS, Knox C, Guo AC, Eisner R, Young N, Gautam B et al. HMDB: knowledgebase for the human metabolome. Nucleic Acids Res 2009; 37: D603-D610.

35. Szklarczyk D, Franceschini A, Kuhn M, Simonovic M, Roth A, Minguez $P$ et al. The STRING database in 2011: functional interaction networks of proteins, globally integrated and scored. Nucleic Acids Res 2011; 39: D561-D568

36. Kastenmüller G, Römisch-Margl W, Wägele B, Altmaier E, Suhre K. metaP-Server: a web-based metabolomics data analysis tool. J Biomed Biotechnol 2011; 2011 (article ID 839862).

37. Huang DW, Sherman BT, Lempicki RA. Systematic and integrative analysis of large gene lists using DAVID bioinformatics resources. Nat Protoc 2009; 4: 44-57.

38. Berardini TZ, Khodiyar VK, Lovering RC, Talmud P. The gene ontology in 2010: extensions and refinements. Nucleic Acids Res 2010; 38: D331-D335.

39. Kanehisa M, Goto S, Furumichi M, Tanabe M, Hirakawa M. KEGG for representation and analysis of molecular networks involving diseases and drugs. Nucleic Acids Res 2010; 38: D355-D360

40. Kampstra P. Beanplot: a boxplot alternative for visual comparison of distributions. J Stat Software Code Snippets 2008; 28: 1-9.

41. Macciardi F, Lucca A, Catalano M, Marino C, Zanardi R, Smeraldi E. Amino acid patterns in schizophrenia: some new findings. Psychiatry Res 1990; 32: 63-70.

42. Yanik M, Vural H, Kocyigit A, Tutkun $\mathrm{H}$, Zoroglu SS, Herken $\mathrm{H}$ et al. Is the arginine-nitric oxide pathway involved in the pathogenesis of schizophrenia? Neuropsychobiology 2003; 47: 61-65.

43. Bernstein H-G, Bogerts B, Keilhoff G. The many faces of nitric oxide in schizophrenia. A review. Schizophr Res 2005; 78: 69-86.

44. Das I, Khan NS, Puri BK, Werge T, Børglum AD, Mors $O$ et al. Elevated platelet calcium mobilization and nitric oxide synthase activity may reflect abnormalities in schizophrenic brain. Biochem Biophys Res Commun 1995; 212: 375-380.

45. Herken H, Uz E, Özyurt H, Akyol Ö. Red blood cell nitric oxide levels in patients with schizophrenia. Schizophr Res 2001; 52: 289-290.

46. Steinberg S, de Jong S, Andreassen OA, Werge T, Børglum AD, Mors $O$ et al. Common variants at VRK2 and TCF4 conferring risk of schizophrenia. Hum Mol Genet 2011; 20: 4076-4081.

47. Middleton FA, Mirnics K, Pierri JN, Lewis DA, Levitt P. Gene expression profiling reveals alterations of specific metabolic pathways in schizophrenia. J Neurosci 2002; 22: 2718-2729. 
48. Tortorella A, Monteleone P, Fabrazzo M, Viggiano A, De Luca L, Maj M. Plasma concentrations of amino acids in chronic schizophrenics treated with clozapine. Neuropsychobiology 2001; 44: 167-171.

49. Carl GF, Brogan MP, Young BK. Is plasma serine a marker for psychosis? Biol Psychiatry 1992; 31: 1130-1135.

50. Fernández-Novoa L, Cacabelos R. Histamine function in brain disorders. Behav Brain Res 2001; 124: 213-233.

51. Prell GD, Green JP, Kaufmann CA, Khandelwal JK, Morrishow AM, Kirch DG et al. Histamine metabolites in cerebrospinal fluid of patients with chronic schizophrenia: their relationships to levels of other aminergic transmitters and ratings of symptoms. Schizophr Res 1995; 14: 93-104.

52. Kenji H, Eiji S, Conny N, Leif L, Masaomi I. Elevated glutamine/glutamate ratio in cerebrospinal fluid of first episode and drug naive schizophrenic patients. BMC Psychiatry 2005; 5: 6 .

53. Burbaeva GS, Boksha IS, Turishcheva MS, Vorobyeva EA, Savushkina OK, Tereshkina EB. Glutamine synthetase and glutamate dehydrogenase in the prefrontal cortex of patients with schizophrenia. Prog Neuro Psychopharmacol Biol Psychiatry 2003; 27: 675-680.

54. Stone JM, Morrison PD, Pilowsky LS. Review: glutamate and dopamine dysregulation in schizophrenia - a synthesis and selective review. J Psychopharmacol 2007; 21: 440-452.

55. Smith QR. Transport of glutamate and other amino acids at the blood-brain barrier. J Nutr 2000; 130: 1016S-1022SS.

56. Fenton WS, Hibbeln J, Knable M. Essential fatty acids, lipid membrane abnormalities, and the diagnosis and treatment of schizophrenia. Biol Psychiatry 2000; 47: 8-21.

57. Horrobin DF, Glen Al, Vaddadi K. The membrane hypothesis of schizophrenia. Schizoph Res 1994; 13: 195-207.
58. Yao JK, Leonard S, Reddy RD. Membrane phospholipid abnormalities in postmortem brains from schizophrenic patients. Schizophr Res 2000; 42: 7-17.

59. Farooqui AA, Horrocks LA. Plasmalogens phospholipase $A_{2}$, and docosahexaenoic acid turnover in brain tissue. JMN 2001; 16: 263-272.

60. Gattaz WF, Köllisch M, Thuren T, Virtanen JA, Kinnunen PKJ. Increased plasma phospholipase-A2 activity in schizophrenic patients: reduction after neuroleptic therapy. Biol Psychiatry 1987; 22: 421-426.

61. Gattaz WF, Hübner CV, Nevalainen TJ, Thuren T, Kinnunen PK. Increased serum phospholipase A2 activity in schizophrenia: a replication study. Biol Psychiatry 1990; 28: 495-501.

62. Gattaz WF, Brunner J, Schmitt A, Maras A. Accelerated breakdown of membrane phospholipids in schizophrenia-implications for the hypofrontality hypothesis. Fortschr Neurol Psychiatr 1994; 62: 489-496.

63. Crisafulli C, Chiesa A, Han C, Lee SJ, Park MH, Balzarro B et al. Case-control association study for 10 genes in patients with schizophrenia: influence of $5 \mathrm{HTR} 1 \mathrm{~A}$ variation rs10042486 on schizophrenia and response to antipsychotics. Eur Arch Psychiatry Clin Neurosci 2011; 262: 199-205.

Translational Psychiatry is an open-access journal published by Nature Publishing Group. This work is licensed under the Creative Commons Attribution-NonCommercial-No Derivative Works 3.0 Unported License. To view a copy of this license, visit http://creativecommons.org/licenses/by-nc-nd/3.0/

Supplementary Information accompanies the paper on the Translational Psychiatry website (http://www.nature.com/tp) 